Jialin Wang, Junbo Zhang, Zhuo Chen* and Lin He

\title{
Hellinger-Reissner variational principle for a class of specified stress problems
}

https://doi.org/10.1515/ijnsns-2021-0039

Received January 29, 2021; accepted November 18, 2021; published online January 31, 2022

\begin{abstract}
Aiming at the problem of the specified stress condition in a partial region of the structure, the Hellinger-Reissner $(\mathrm{H}-\mathrm{R})$ variational principle is studied to provide a theoretical basis for the finite element numerical analysis. By introducing the unknown non-elastic strain as an additional unknown quantity to fulfill the specified stress condition, the elastic mechanics governing equations for the specified stress problem are given. Both stress and unknown non-elastic strain are taken as independent variables to establish the complementary energy principle and virtual work equation which are equivalent to the elastic mechanical control equation of the specified stress problem. Based on the conventional $\mathrm{H}-\mathrm{R}$ variational principle, using displacement, stress and unknown non-elastic strain as independent variables, a $\mathrm{H}-\mathrm{R}$ variational functional that satisfies the specified stress conditions is established by using Lagrange multiplier method. Also the variational functional with displacement, elastic strain and unknown non-elastic strain as independent variables is deduced by transforming the stress into the elastic strain. The corresponding finite formulae are derived based on an intra-element stress hybridization method. The $\mathrm{H}-\mathrm{R}$ variational principle for the specified stress problem takes non-elastic strain as an independent variable, so that the stress explicitly appears in the equilibrium equation of the element and structure, which expands the application range and capabilities of the existing variational principle and finite element method. The correctness and accuracy of the theory and algorithm are verified by numerical examples.
\end{abstract}

Keywords: finite element methods; Hellinger-Reissner variational principle; non-elastic; specified stress problem.

\section{Introduction}

The independent variables of conventional elastic mechanics problems are displacement, strain and stress, which are solved by governing equations, displacement boundary conditions and force boundary conditions. The factors that affect the mechanical response of the elastomer, such as temperature changes, initial stresses, and initial strains, must be known as prescribed values, and the stress and internal force of the elastomer cannot be controlled in advance. Specifically, under the definite conditions of elastic mechanics, the factors affecting and adjusting the stress do not be regarded as unknown quantities in the conventional finite element method, whether it is the displacement method or the mixed variable with stress as the independent variable. The stress is not adjustable and impossible to be set constraints at the specified region in the domain.

In engineering practice, there is a class of specified stress problem, which can be affected by some factors or regulatory measures to make the stress or internal force of certain parts of the structure meet the specified

\footnotetext{
*Corresponding author: Zhuo Chen, Chongqing Jiaotong University, NO.66 Xuefu Road, 400074, Chongqing, China, E-mail: 82517863@qq.com

Jialin Wang, Junbo Zhang and Lin He, Chongqing Jiaotong University, NO.66 Xuefu Road, 400074, Chongqing, China, E-mail:747085700@qq.com (J. Wang),84375981@qq.com (. Zhang), 809682248@qq.com (L. He)
} 
constraint conditions, such as: (1) The stiffness and known stress of the pre-stressed tendons in the stressed concrete structure need be simulated simultaneously; (2) The cable-stayed bridge needs to have specified tension values of partial/all cables through cables length adjustment to ensure the smooth linear shape and uniform internal force of main beam in the completed state of the bridge; (3) The stress at the crack interface is zero after cracking; (4) In some engineering problems, the measured current stress of the structure is used to invert unknown influencing factors.

It should be stressed that the specified stress problem is different from the initial stress problem. The difference between the two is as follows: (1) In the initial stress problem, the initial stress is the initial value before loading. After the structure is deformed under load, the stress evolves on the basis of the initial value, and the final value of the stress cannot be specified as constraint conditions; (2) In the specified stress problem, there are certain influencing factors and control measures that make the stress of the structure after the load have a specified value (known or need to achieve), that is, the stress in the calculated result is required to be equal to the specified value.

The finite element method is an important method to obtain numerical solutions of complex problems. The stress in the displacement based finite element method suffers low accuracy from its calculation via derivation of displacement field.

Based on the classical or modified $\mathrm{H}-\mathrm{R}$ variational principle, the hybrid stress element and hybrid(mixed) element are used to discretize the stress and displacement fields, then the equilibrium equations and stress boundary conditions could be better fulfilled, the lock-in(locking) phenomenon could be avoided, and high stress accuracy could be achieved [1, 2]. Due to such calculation features, the finite element method based on the $\mathrm{H}-\mathrm{R}$ variational principle has been paid attention to by many scholars. Lei et al. [3] derived the stress optimization conditions of the strain gradient hybrid element based on the $\mathrm{H}-\mathrm{R}$ variational principle and the couple stress theory, and constructed a planar 4-node hybrid element. Numerical examples show that reasonable numerical results for both compressible and incompressible gradient materials can be achieved via the proposed element.

Considering the coupling of axial stress and bending moment naturally, Auricchio et al. $[4,5]$ established a non-prismatic beam element based on the $\mathrm{H}-\mathrm{R}$ variational principle. The accuracy of the presented element is consistent with the analysis of the solid element. Mohammad and Nima [6] used independent interpolation of force and displacement to establish curved beam elements that can consider radial edge cracks, eliminating the phenomenon of shear locking and showing good performance in static force, free vibration and stress intensity factor calculation. D'Ottavio [7] and Altay [8] used the generalized $\mathrm{H}-\mathrm{R}$ hybrid variational principle to calculate the laminated structure of piezoelectric materials. Lee et al. [9] used the $\mathrm{H}-\mathrm{R}$ variational principle to improve the MITC3 shell element and increase the convergence.

Domínguez et al. [10] used the $\mathrm{H}-\mathrm{R}$ variational principle to establish a linear homogeneous shell element suitable for thin-to-medium thickness. Numerical examples show that it is more accurate than conventional shell elements and MITC6 shell elements, and the elastodynamic behavior of thick shells is further discussed [11]. Sangtarash et al. [12] established a 4-node flat shell element based on the H-R variational principle by using the analytical solution of the Airy stress function in the Cartesian coordinate system as a trial function. Numerical examples have verified its good performance and can adapt to complex geometries and boundary conditions. ScH-Röder et al. [13] used the Prange-Hellinger-Reissner generalized variational principle to deal with the small deformation elastoplastic problem. Viebahn et al. [14, 15] constructed an element based on the $\mathrm{H}-\mathrm{R}$ variational principle, which overcomes the self-locking of linear elastic incompressible materials and is used to simulate the mechanical behavior of hyperelastic materials.

Artioli et al. [16] and Dassi et al. [17] studied the virtual element method based on the $\mathrm{H}-\mathrm{R}$ variational principle to deal with plane and three-dimensional problems. Dong Yi et al. [18] used the H-R variational principle to introduce the hybrid element idea into the natural element method, and used the Sibson method to interpolate the stress, and established a hybrid natural element method to improve the accuracy of stress; Ma et al. $[19,20]$ studied the large deformation of elastic and elastoplastic materials by using hybrid natural element method based on the incremental $\mathrm{H}-\mathrm{R}$ variational principle of T.L. format. Numerical examples show that hybrid natural element method is more accurate and faster than conventional natural element method. 
Yang et al. [21] constructed a hybrid generalized element based on the H-R variational principle, which can improve accuracy and the order of the element displacement field and stress field without increasing the number of element nodes.

Ding et al. [22] established a hybrid element for geometric and material nonlinear analysis based on the $\mathrm{H}-\mathrm{R}$ variation. The fiber section model is applied to the numerical analysis of concrete-filled steel tubular (CFST) members, which has good computational efficiency and numerical stability. Qing et al. [23] interpolated displacement and stress independently based on the principle of minimum potential energy and $\mathrm{H}-\mathrm{R}$ variational principle, and established a mixed element with displacement and stress as unknown quantities, which avoided zeros on the main diagonal of the coefficient matrix in the traditional mixing method and can satisfy the stress boundary conditions better. Zhao et al. [24] improved the integration method of literature [23], and increased the calculation speed by combining the enhanced hypothetical strain theory.

Qing et al. [25] considered general three-dimensional problems, based on the modified $\mathrm{H}-\mathrm{R}$ variational principle and the symplectic theory of elastic mechanics, established a block mixed isoparametric element containing out-of-plane stress and displacement variables. Numerical examples proved that the convergence equilibrium, stability and high precision of the calculation. The convergence rate of the stress variable is close to the traditional 20-node displacement coordination reduction integral element. Liu et al. [26] established a parameter-containing, non-compatible sympletic element to solve the static problem of the steady-state temperature field based on the non-compatible symplectic element theory and the generalized $\mathrm{H}-\mathrm{R}$ variational principle, and applied it to the stress analysis of the thermoelastic composite laminate. The result is stable and high precision. Based on the principle of minimum potential energy and $\mathrm{H}-\mathrm{R}$ variational principle, Yao et al. [27] constructed a 20-node hexahedral symplectic element containing displacement and three out-of-plane stress variables, which is not limited by the element thickness and structural geometry. Numerical examples show that the results of this element have stable convergence and high accuracy.

Dujc et al. [28] constructed a hybrid quadrilateral element with discrete cracks (interfaces) based on the $\mathrm{H}-\mathrm{R}$ principle by embedding discontinuities in the displacement. Numerical examples demonstrate that the element has good crack tracking capabilities. He [29] gave a way to establish the variational principle through the semi-inverse method and applied this method to the thermodynamically coupled finite displacement problem [30]. This method uses the trial function to derive the HR variational principle directly without using the Lagrange multiplier method. Cannarozzi et al. [31] also deal with linear quasi-static thermodynamics based on the generalized $\mathrm{H}-\mathrm{R}$ variational principle with independent variables of displacement, stress, temperature and heat flux.

The above references indicate that a variety of high-precision numerical methods can be established based on the $\mathrm{H}-\mathrm{R}$ variational principle, but the current $\mathrm{H}-\mathrm{R}$ variational principle does not consider the specified stress problem, and it is difficult to make the stress meet the specified condition in the result.

\section{The governing equations for specified stress problems}

A problem of the specified stress condition by adjusting the stress through non-elastic strain is discussed. Using the complementary energy functional $B\left(\sigma_{i j}\right)$, the governing equation of the specified stress problem in elastic mechanics can be expressed as:

In the elastomer domain $V$ :

$$
\begin{aligned}
& \sigma_{i j, j}+f_{i}=0 \\
& \frac{\partial B\left(\sigma_{i j}\right)}{\partial \sigma_{i j}}=\varepsilon_{i j}^{e}
\end{aligned}
$$

Within the specified stress domain $V_{\sigma}$ :

$$
\begin{aligned}
\sigma_{i j} & =\sigma_{i j}^{0} \\
\varepsilon_{i j}^{e}+\varepsilon_{i j}^{0}+\varepsilon_{i j}^{p} & =\frac{1}{2}\left(u_{i, j}+u_{j, i}\right)
\end{aligned}
$$


In the domain $V_{u}=V-V_{\sigma}$ without specified stress,

$$
\varepsilon_{i j}^{e}+\varepsilon_{i j}^{p}=\frac{1}{2}\left(u_{i, j}+u_{j, i}\right)
$$

On the specified displacement boundary $S_{u}$

$$
u_{i}=\bar{u}_{i}
$$

On the specified stress boundary $S_{\sigma}$

$$
\sigma_{i j} n_{j}=p_{i}
$$

In the above formula, $\sigma_{i j}$ is stress, $u_{i}$ is displacement, $\varepsilon_{i j}^{e}$, $\varepsilon_{i j}^{p}$, $\varepsilon_{i j}^{0}$ are elastic strain, known non-elastic strain and unknown non-elastic strain respectively, $B\left(\sigma_{i j}\right)$ is elastic complementary energy functional expressed by stress $\sigma_{i j}$, $u_{i, j}=\partial u_{i} / \partial x_{j}, f_{i}$ and $p_{i}$ are the concentration of body force and surface force, $\bar{u}_{i}$ is the specified displacement of the boundary, $\sigma_{i j}^{0}$ is the specified stress value, elastic. The solution domain of elastomer problem is denoted as $V=V_{\sigma}+V_{u}$, and the boundary of the solution domain is denoted as $S=S_{u}+S_{\sigma}, n_{i}$ is the unit vector of the outer normal direction of the boundary with the subscriptsi, $j=1,2,3$.

$\varepsilon_{i j}^{0}$ in formula (4) is the adjustment factor for achieving the specified condition of formula (3); Without adjustment factor be introduced, the stress is determined by formulas (1), (2), (5)-(7) collectively, additional specified conditions cannot be introduced. If $\varepsilon_{i j}^{0}=0$ and $\sigma_{i j}^{0}$ is taken as an unknown quantity (that is, Eq. (3) is invalidated), then formulas (1)-(7) degenerate into the governing equations of classical elasticity.

\section{Complementary energy principle of specified stress problem}

The complementary energy functional of the classical elasticity problem can be expressed as:

$$
\Pi_{C}\left(\sigma_{i j}\right)=\int_{V} B\left(\sigma_{i j}\right) \mathrm{d} V-\int_{S_{u}} \sigma_{i j} n_{j} \bar{u}_{i} \mathrm{~d} S
$$

With variation:

$$
\delta \Pi_{\mathrm{C}}\left(\sigma_{i j}\right)=\delta \int_{V} B\left(\sigma_{i j}\right) \mathrm{d} V-\delta \int_{S_{u}} \sigma_{i j} n_{j} \bar{u}_{i} \mathrm{~d} S
$$

Here

$$
\begin{gathered}
\delta \int_{V} B\left(\sigma_{i j}\right) \mathrm{d} V=\int_{V} \frac{\partial B\left(\sigma_{i j}\right)}{\partial \sigma_{i j}} \delta \sigma_{i j} \mathrm{~d} V \\
\delta \int_{S_{u}} \sigma_{i j} n_{j} \bar{u}_{i} \mathrm{~d} S=\int_{S_{u}} \delta \sigma_{i j} n_{j} \bar{u}_{i} \mathrm{~d} S
\end{gathered}
$$

Note that $\delta \sigma_{i j, j}=0$ in the domain $V$ and $\delta \sigma_{i j} n_{j}=0$ on the stress boundary $S_{\sigma}$, so

$$
\int_{V} u_{i, j} \delta \sigma_{i j} \mathrm{~d} V=\int_{V}\left(u_{i} \delta \sigma_{i j}\right)_{, j} \mathrm{~d} V-\int_{V} u_{i} \delta \sigma_{i j, j} \mathrm{~d} V=\int_{S_{u}} \delta \sigma_{i j} n_{j} u_{i} \mathrm{~d} S
$$

Substituting formulas (10)-(12) into formula (9), it can be obtained

$$
\begin{aligned}
\delta \Pi_{\mathrm{C}}\left(\sigma_{i j}\right) & =\int_{V} \frac{\partial B\left(\sigma_{i j}\right)}{\partial \sigma_{i j}} \delta \sigma_{i j} \mathrm{~d} V-\int_{V} u_{i, j} \delta \sigma_{i j} \mathrm{~d} V+\int_{S_{u}} u_{i} \delta \sigma_{i j} n_{j} \mathrm{~d} S-\int_{S_{u}} \delta \sigma_{i j} n_{j} \bar{u}_{i} \mathrm{~d} S \\
& =\int_{V}\left[\frac{\partial B\left(\sigma_{i j}\right)}{\partial \sigma_{i j}}-\frac{1}{2}\left(u_{i, j}+u_{j, i}\right)\right] \delta \sigma_{i j} \mathrm{~d} V+\int_{S_{u}} \delta \sigma_{i j} n_{j}\left(u_{i}-\bar{u}_{i}\right) \mathrm{d} S
\end{aligned}
$$


For the specified stress problem, using the Lagrange multiplier method to establish a conditional functional:

$$
\Pi_{\mathrm{CS}}\left(\sigma_{i j}, \lambda_{i j}\right)=\int_{V} B\left(\sigma_{i j}\right) \mathrm{d} V-\int_{S_{u}} \sigma_{i j} n_{j} \bar{u}_{i} \mathrm{~d} S+\int_{V_{\sigma}}\left(\sigma_{i j}-\sigma_{i j}^{0}\right) \lambda_{i j} \mathrm{~d} V
$$

Variation of last part of formula (14)

$$
\delta \int_{V_{\sigma}}\left(\sigma_{i j}-\sigma_{i j}^{0}\right) \lambda_{i j} \mathrm{~d} V=\int_{V_{\sigma}} \delta \sigma_{i j} \lambda_{i j} \mathrm{~d} V+\int_{V_{\sigma}}\left(\sigma_{i j}-\sigma_{i j}^{0}\right) \delta \lambda_{i j} \mathrm{~d} V
$$

Substituting formulas (13) and (15) into formula (14), it can be obtained

$$
\begin{aligned}
\delta \Pi_{\mathrm{CS}}\left(\sigma_{i j}, \lambda_{i j}\right)= & \int_{V_{\sigma}}\left(\sigma_{i j}-\sigma_{i j}^{0}\right) \delta \lambda_{i j} \mathrm{~d} V+\int_{S_{u}} \delta \sigma_{i j} n_{j}\left(u_{i}-\bar{u}_{i}\right) \mathrm{d} S \\
& +\int_{V_{u}}\left[\frac{\partial B\left(\sigma_{i j}\right)}{\partial \sigma_{i j}}-\frac{1}{2}\left(u_{i, j}+u_{j, i}\right)\right] \delta \sigma_{i j} \mathrm{~d} V+\int_{V_{\sigma}}\left[\frac{\partial B\left(\sigma_{i j}\right)}{\partial \sigma_{i j}}+\lambda_{i j}-\frac{1}{2}\left(u_{i, j}+u_{j, i}\right)\right] \delta \sigma_{i j} \mathrm{~d} V
\end{aligned}
$$

$\lambda_{i j}$ is a non-elastic strain, reset as $\varepsilon_{i j}^{0}$, so the complementary energy functional of the specified stress problem is:

$$
\Pi_{\mathrm{CS}}\left(\sigma_{i j}, \varepsilon_{i j}^{0}\right)=\int_{V} B\left(\sigma_{i j}\right) \mathrm{d} V-\int_{S_{u}} \sigma_{i j} n_{j} \bar{u}_{i} \mathrm{~d} S+\int_{V_{\sigma}}\left(\sigma_{i j}-\sigma_{i j}^{0}\right) \varepsilon_{i j}^{0} \mathrm{~d} V
$$

If there is a known non-elastic strain $\varepsilon_{i j}^{p}$, the complementary energy functional can be further expressed as

$$
\Pi_{\mathrm{CS}}\left(\sigma_{i j}, \varepsilon_{i j}^{0}\right)=\int_{V} B\left(\sigma_{i j}\right) \mathrm{d} V-\int_{S_{u}} \sigma_{i j} n_{j} \bar{u}_{i} \mathrm{~d} S+\int_{V_{\sigma}}\left(\sigma_{i j}-\sigma_{i j}^{0}\right) \varepsilon_{i j}^{0} \mathrm{~d} V+\int_{V} \sigma_{i j} \varepsilon_{i j}^{p} \mathrm{~d} V
$$

Using formula (2), variation of above equation can be obtained:

$$
\begin{aligned}
\delta \Pi_{\mathrm{CS}}\left(\sigma_{i j}, \varepsilon_{i j}^{0}\right)= & \int_{V_{\sigma}}\left(\sigma_{i j}-\sigma_{i j}^{0}\right) \delta \varepsilon_{i j}^{0} \mathrm{~d} V+\int_{S_{u}} \delta \sigma_{i j} n_{j}\left(u_{i}-\bar{u}_{i}\right) \mathrm{d} S \\
& +\int_{V_{u}}\left[\varepsilon_{i j}^{e}+\varepsilon_{i j}^{p}-\frac{1}{2}\left(u_{i, j}+u_{j, i}\right)\right] \delta \sigma_{i j} \mathrm{~d} V+\int_{V_{\sigma}}\left[\varepsilon_{i j}^{e}+\varepsilon_{i j}^{p}+\varepsilon_{i j}^{0}-\frac{1}{2}\left(u_{i, j}+u_{j, i}\right)\right] \delta \sigma_{i j} \mathrm{~d} V
\end{aligned}
$$

According to the arbitrariness of $\delta \sigma_{i j}$ and $\delta \varepsilon_{i j}^{0}$, formulas (3)-(6) can be obtained by $\delta \Pi_{\mathrm{CS}}\left(\sigma_{i j}, \varepsilon_{i j}^{0}\right)=0$.

The complementary energy theorem for the specified stress problem can be expressed as: among all possible static stress $\sigma_{i j}$ and all possible non-elastic strains $\varepsilon_{i j}^{0}$ that satisfy formulas (1), (2) and (7), the true $\sigma_{i j}$ and $\varepsilon_{i j}^{0}$ makes the complementary energy functional (18) take a stationary value, that is, the solution of $\delta \Pi_{\mathrm{CS}}\left(\sigma_{i j}, \varepsilon_{i j}^{0}\right)=0$ is equivalent to the solution of the governing Eqs. (3)-(6) of the specified stress problem.

According to formula (18), the virtual work equation corresponding to the complementary energy principle of the specified stress problem can also be obtained:

$$
\int_{V} \frac{\partial B\left(\sigma_{i j}\right)}{\partial \sigma_{i j}} \delta \sigma_{i j} \mathrm{~d} V+\int_{V_{\sigma}} \delta \sigma_{i j} \varepsilon_{i j}^{0} \mathrm{~d} V+\int_{V_{\sigma}}\left(\sigma_{i j}-\sigma_{i j}^{0}\right) \delta \varepsilon_{i j}^{0} \mathrm{~d} V+\int_{V} \delta \sigma_{i j} \varepsilon_{i j}^{p} \mathrm{~d} V=\int_{S_{u}} \delta \sigma_{i j} n_{j} \bar{u}_{i} \mathrm{~d} S
$$




\section{Hellinger-Reissner variational principle for specified stress problems}

The functional of $\mathrm{H}-\mathrm{R}$ variational principle of classical elasticity problem [2] is:

$$
\Pi_{\mathrm{HR}}\left(\sigma_{i j}, u_{i}\right)=\int_{V} B\left(\sigma_{i j}\right) \mathrm{d} V+\int_{V}\left(\sigma_{i j, j}+f_{i}\right) u_{i} \mathrm{~d} V-\int_{S_{u}} \sigma_{i j} n_{j} \bar{u}_{i} \mathrm{~d} S-\int_{S_{\sigma}}\left(\sigma_{i j} n_{j}-p_{i}\right) u_{i} \mathrm{~d} S
$$

From the divergence theorem:

$$
\int_{V} \sigma_{i j, j} u_{i} \mathrm{~d} V=\int_{S_{\sigma}+S_{u}} \sigma_{i j} n_{j} u_{i} \mathrm{~d} S-\int_{V} \sigma_{i j} u_{i, j} \mathrm{~d} V
$$

Therefore, the functional of the $\mathrm{H}-\mathrm{R}$ variational principle can be rewritten as:

$$
\Pi_{\mathrm{HR}}\left(\sigma_{i j}, u_{i}\right)=\int_{V}\left[B\left(\sigma_{i j}\right)-\sigma_{i j} u_{i, j}+f_{i} u_{i}\right] \mathrm{d} V+\int_{S_{u}} \sigma_{i j} n_{j}\left(u_{i}-\bar{u}_{i}\right) \mathrm{d} S+\int_{S_{\sigma}} p_{i} u_{i} \mathrm{~d} S
$$

Variation can be obtained:

$$
\begin{aligned}
\delta \Pi_{\mathrm{HR}}\left(\sigma_{i j}, u_{i}\right)= & \int_{V}\left[\frac{\partial B\left(\sigma_{i j}\right)}{\partial \sigma_{i j}}-\frac{1}{2}\left(u_{i, j}+u_{j, i}\right)\right] \delta \sigma_{i j} \mathrm{~d} V+\int_{V}\left(\sigma_{i j, j}+f_{i}\right) \delta u_{i} \mathrm{~d} V \\
& +\int_{S_{\sigma}}\left(p_{i}-\sigma_{i j} n_{j}\right) \delta u_{i} \mathrm{~d} S+\int_{S_{u}} \delta \sigma_{i j} n_{j}\left(u_{i}-\bar{u}_{i}\right) \mathrm{d} S
\end{aligned}
$$

The geometric equations, equilibrium equations, stress boundary conditions and displacement boundary conditions of elasticity can be obtained by $\delta \Pi_{\mathrm{HR}}\left(\sigma_{i j}, u_{i}\right)=0$. Therefore, the $\mathrm{H}-\mathrm{R}$ variational principle of classical elasticity problems can be expressed as: among all stresses and displacements satisfying formula (2), the true stress $\sigma_{i j}$ and displacement $u_{i}$ make the functional $\Pi_{\mathrm{HR}}\left(\sigma_{i j}, u_{i}\right)$ take a stationary value.

On the basis of the conventional $\mathrm{H}-\mathrm{R}$ variational principle, the conditional functional is established by the Lagrange multiplier method:

$$
\begin{aligned}
\Pi_{\mathrm{HRS}}\left(\sigma_{i j}, u_{i}, \lambda_{i j}\right)= & \int_{V}\left[B\left(\sigma_{i j}\right)-\sigma_{i j} u_{i, j}+f_{i} u_{i}\right] \mathrm{d} V+\int_{S_{u}} \sigma_{i j} n_{j}\left(u_{i}-\bar{u}_{i}\right) \mathrm{d} S+\int_{S_{\sigma}} p_{i} u_{i} \mathrm{~d} S \\
& +\int_{V_{\sigma}}\left(\sigma_{i j}-\sigma_{i j}^{0}\right) \lambda_{i j} \mathrm{~d} V
\end{aligned}
$$

Substituting Eqs. (15) and (24) into Eq. (25):

$$
\begin{aligned}
\delta \Pi_{\mathrm{HRS}}\left(\sigma_{i j}, u_{i}, \lambda_{i j}\right)= & \int_{V}\left(\sigma_{i j, j}+f_{i}\right) \delta u_{i} \mathrm{~d} V+\int_{V_{\sigma}}\left(\sigma_{i j}-\sigma_{i j}^{0}\right) \delta \lambda_{i j} \mathrm{~d} V \\
& +\int_{V_{u}}\left[\frac{\partial B\left(\sigma_{i j}\right)}{\partial \sigma_{i j}}-\frac{1}{2}\left(u_{i, j}+u_{j, i}\right)\right] \delta \sigma_{i j} \mathrm{~d} V+\int_{V_{\sigma}}\left[\frac{\partial B\left(\sigma_{i j}\right)}{\partial \sigma_{i j}}+\lambda_{i j}-\frac{1}{2}\left(u_{i, j}+u_{j, i}\right)\right] \delta \sigma_{i j} \mathrm{~d} V \\
& +\int_{S_{\sigma}}\left(p_{i}-\sigma_{i j} n_{j}\right) \delta u_{i} \mathrm{~d} S+\int_{S_{u}} \delta \sigma_{i j} n_{j}\left(u_{i}-\bar{u}_{i}\right) \mathrm{d} S
\end{aligned}
$$

$\lambda_{i j}$ is a non-elastic strain, reset as $\varepsilon_{i j}^{0}$, so the functional of the $\mathrm{H}-\mathrm{R}$ variational principle of the specified stress problem is obtained as: 


$$
\begin{aligned}
\Pi_{\mathrm{HRS}}\left(\sigma_{i j}, u_{i}, \varepsilon_{i j}^{0}\right)= & \int_{V}\left[B\left(\sigma_{i j}\right)-\sigma_{i j} u_{i, j}+f_{i} u_{i}\right] \mathrm{d} V+\int_{S_{u}} \sigma_{i j} n_{j}\left(u_{i}-\bar{u}_{i}\right) \mathrm{d} S+\int_{S_{\sigma}} p_{i} u_{i} \mathrm{~d} S \\
& +\int_{V_{\sigma}}\left(\sigma_{i j}-\sigma_{i j}^{0}\right) \varepsilon_{i j}^{0} \mathrm{~d} V
\end{aligned}
$$

If there is a known non-elastic strain $\varepsilon_{i j}^{p}$, the functional of the $\mathrm{H}-\mathrm{R}$ variational principle for the specified stress problem can be further expressed as:

$$
\begin{aligned}
\Pi_{\mathrm{HRS}}\left(\sigma_{i j}, u_{i}, \varepsilon_{i j}^{0}\right)= & \int_{V}\left[B\left(\sigma_{i j}\right)-\sigma_{i j} u_{i, j}+f_{i} u_{i}\right] \mathrm{d} V+\int_{S_{u}} \sigma_{i j} n_{j}\left(u_{i}-\bar{u}_{i}\right) \mathrm{d} S+\int_{S_{\sigma}} p_{i} u_{i} \mathrm{~d} S \\
& +\int_{V_{\sigma}}\left(\sigma_{i j}-\sigma_{i j}^{0}\right) \varepsilon_{i j}^{0} \mathrm{~d} V+\int_{V} \sigma_{i j} \varepsilon_{i j}^{p} \mathrm{~d} V
\end{aligned}
$$

Using formula (2), variation of above equation can be obtained:

$$
\begin{aligned}
\delta \Pi_{\mathrm{HRS}}\left(\sigma_{i j}, u_{i}, \varepsilon_{i j}^{0}\right)= & \int_{V}\left(\sigma_{i j, j}+f_{i}\right) \delta u_{i} \mathrm{~d} V+\int_{V_{\sigma}}\left(\sigma_{i j}-\sigma_{i j}^{0}\right) \delta \lambda_{i j} \mathrm{~d} V \\
& +\int_{V_{u}}\left[\varepsilon_{i j}^{e}+\varepsilon_{i j}^{p}-\frac{1}{2}\left(u_{i, j}+u_{j, i}\right)\right] \delta \sigma_{i j} \mathrm{~d} V+\int_{V_{\sigma}}\left[\varepsilon_{i j}^{e}+\varepsilon_{i j}^{p}+\varepsilon_{i j}^{0}-\frac{1}{2}\left(u_{i, j}+u_{j, i}\right)\right] \delta \sigma_{i j} \mathrm{~d} V \\
& +\int_{S_{\sigma}}\left(p_{i}-\sigma_{i j} n_{j}\right) \delta u_{i} \mathrm{~d} S+\int_{S_{u}} \delta \sigma_{i j} n_{j}\left(u_{i}-\bar{u}_{i}\right) \mathrm{d} S
\end{aligned}
$$

According to the arbitrariness of $\delta \sigma_{i j}, \delta u_{i}$ and $\delta \varepsilon_{i j}^{0}$, it can be known that $\delta \Pi_{\text {HRS }}\left(\sigma_{i j}, u_{i}, \varepsilon_{i j}^{0}\right)=0$ is equivalent to the elastic mechanics governing equation of the specified stress problem. Therefore, the $\mathrm{H}-\mathrm{R}$ variational principle of the specified stress problem can be expressed as: among all the stress $\sigma_{i j}$, non-elastic strain $\varepsilon_{i j}^{0}$ and the displacement $u_{i}$ which satisfy formula (2), the true stress $\sigma_{i j}$, displacement $u_{i}$ and non-elastic strain $\varepsilon_{i j}^{0}$ makes the functional $\Pi_{\mathrm{HRS}}\left(\sigma_{i j}, u_{i}, \varepsilon_{i j}^{0}\right)$ in Eq. (28) take a stationary value.

\section{Hybrid stress element formulation based on the $H-R$ variational principle of the specified stress problem}

\subsection{Variational functional}

Considering that the specified stress problem contains known non-elastic strains and unknown non-elastic strains, the independent stress function $\sigma_{i j}$ in Eq. (28) is replaced with elastic strain $\varepsilon_{i j}^{e}$ for linear elastic materials, and the negative value of the functional is used Voigt notation is written as:

$$
\begin{aligned}
\Pi_{\mathrm{HRS}}\left(\boldsymbol{u}, \boldsymbol{\varepsilon}^{\boldsymbol{e}}, \boldsymbol{\varepsilon}^{0}\right)= & \int_{V}\left[-\frac{1}{2}\left(\boldsymbol{\varepsilon}^{\boldsymbol{e}}\right)^{\mathrm{T}} \boldsymbol{C} \boldsymbol{\varepsilon}^{\boldsymbol{e}}+\left(\boldsymbol{C} \boldsymbol{\varepsilon}^{\boldsymbol{e}}\right)^{\mathrm{T}}(\boldsymbol{D} \boldsymbol{u})-\overline{\boldsymbol{f}}^{\mathrm{T}} \boldsymbol{u}\right] \mathrm{d} V-\int_{S_{u}} \boldsymbol{T}^{\mathrm{T}}(\boldsymbol{u}-\overline{\boldsymbol{u}}) \mathrm{d} S \\
& -\int_{S_{\sigma}} \overline{\boldsymbol{T}}^{\mathrm{T}} \boldsymbol{u} \mathrm{d} S-\int_{V_{\sigma}}\left(\boldsymbol{C} \boldsymbol{\varepsilon}^{\boldsymbol{e}}-\boldsymbol{\sigma}^{0}\right)^{\mathrm{T}} \boldsymbol{\varepsilon}^{0} \mathrm{~d} V-\int_{V}\left(\boldsymbol{C} \boldsymbol{\varepsilon}^{\boldsymbol{e}}\right)^{\mathrm{T}} \boldsymbol{\varepsilon}^{\boldsymbol{p}} \mathrm{d} V
\end{aligned}
$$

Where $\boldsymbol{u}$ is the displacement vector, $\varepsilon^{\boldsymbol{e}}, \varepsilon^{0}$ and $\varepsilon^{p}$ are the vector notation of elastic strain, unknown nonelastic strain and known non-elastic strain respectively, $\sigma^{0}$ is the specified stress vector notation, and $\boldsymbol{D}$ is the 
displacement derivative operator matrix, $\boldsymbol{T}$ and $\overline{\boldsymbol{T}}$ represent the unknown and known face force vectors, $\overline{\boldsymbol{f}}$ is the known body force vector, $\overline{\boldsymbol{u}}$ is the boundary vector with specified displacement, $\boldsymbol{C}$ is the modulus matrix, satisfying $\boldsymbol{\sigma}=\boldsymbol{C} \varepsilon^{\boldsymbol{e}}, \boldsymbol{\sigma}$ is vector notation of stress.

For the sake of simplification, specified stress conditions in the entire element are assumed (i.e., $V_{\sigma}=V$; when no stress conditions are specified, just take $\varepsilon^{0}=0$ ). In addition, the displacement interpolation mode is considered if the coordination condition and displacement boundary condition between the elements are satisfied. Then the displacement specified boundary term in Eq. (30) is no need to be considered, that is, $\int_{S_{u}} \boldsymbol{T}^{\mathrm{T}}(\boldsymbol{u}-\overline{\boldsymbol{u}}) \mathrm{d} S \equiv 0$, the functional of formula (30) can be re-expressed as:

$$
\begin{aligned}
\Pi_{\mathrm{HRS}}\left(\boldsymbol{u}, \boldsymbol{\varepsilon}^{\boldsymbol{e}}, \boldsymbol{\varepsilon}^{0}\right)= & \int_{V}\left[\left(\boldsymbol{\varepsilon}^{\boldsymbol{e}}\right)^{\mathrm{T}} \boldsymbol{C}(\boldsymbol{D} \boldsymbol{u})-\frac{1}{2}\left(\boldsymbol{\varepsilon}^{\boldsymbol{e}}\right)^{\mathrm{T}} \boldsymbol{C} \boldsymbol{\varepsilon}^{\boldsymbol{e}}-\left(\boldsymbol{\varepsilon}^{\boldsymbol{e}}\right)^{\mathrm{T}} \boldsymbol{C} \varepsilon^{0}-\left(\boldsymbol{\varepsilon}^{\boldsymbol{e}}\right)^{\mathrm{T}} \boldsymbol{C} \boldsymbol{\varepsilon}^{\boldsymbol{p}}\right] \mathrm{d} V \\
& +\int_{V}\left(\boldsymbol{\sigma}^{0}\right)^{\mathrm{T}} \boldsymbol{\varepsilon}^{0} \mathrm{~d} V-\int_{V} \overline{\boldsymbol{f}}^{\mathrm{T}} \boldsymbol{u} \mathrm{d} V-\int_{S_{\sigma}} \overline{\boldsymbol{T}}^{\mathrm{T}} \boldsymbol{u} \mathrm{d} S
\end{aligned}
$$

\subsection{Specify the finite element formula of the specified stress hybrid element}

The interpolation modes of displacement $\boldsymbol{u}$, total strain $\varepsilon=\varepsilon^{\boldsymbol{e}}+\varepsilon^{0}+\varepsilon^{\boldsymbol{p}}$, and unknown non-elastic strain $\varepsilon^{0}$ are supposed [2]

$$
\begin{gathered}
\boldsymbol{u}=\boldsymbol{N} \boldsymbol{q} \\
\varepsilon=\boldsymbol{P} \boldsymbol{\alpha} \\
\varepsilon^{0}=\boldsymbol{R} \boldsymbol{\beta}
\end{gathered}
$$

Where $\boldsymbol{q}$ is the nodal displacement, $\boldsymbol{\alpha}$ is the interpolation parameter of the total strain, $\boldsymbol{\beta}$ is the interpolation parameter of the unknown non-elastic strain, and $\boldsymbol{N}, \boldsymbol{P}, \boldsymbol{R}$ are the corresponding interpolation matrices.

From Eq. (32), the expression form of displacement derivative can be obtained

$$
D u=D N q=B q
$$

The elastic strain of the element is expressed by the total strain interpolation and the unknown non-elastic strain interpolation as

$$
\varepsilon^{e}=\varepsilon-\varepsilon^{0}-\varepsilon^{p}=P \alpha-R \beta-\varepsilon^{p}
$$

Using three variable interpolation methods, the original functional can be transformed into a functional with various interpolation parameters as variables:

$$
\begin{aligned}
\Pi_{\mathrm{HRS}}(\boldsymbol{q}, \boldsymbol{\alpha}, \boldsymbol{\beta})= & \int_{V}\left[\left(\boldsymbol{P} \boldsymbol{\alpha}-\boldsymbol{R} \boldsymbol{\beta}-\boldsymbol{\varepsilon}^{\boldsymbol{p}}\right)^{\mathrm{T}} \boldsymbol{C}\left(\boldsymbol{B} \boldsymbol{q}-\frac{1}{2}\left(\boldsymbol{P} \boldsymbol{\alpha}+\boldsymbol{R} \boldsymbol{\beta}+\boldsymbol{\varepsilon}^{\boldsymbol{p}}\right)\right)\right] \mathrm{d} V \\
& +\int_{V}\left(\boldsymbol{\sigma}^{0}\right)^{\mathrm{T}} \boldsymbol{R} \boldsymbol{\beta} \mathrm{d} V-\int_{V} \overline{\boldsymbol{f}}^{\mathrm{T}} \boldsymbol{N} \boldsymbol{q} \mathrm{d} V-\int_{S_{\sigma}} \overline{\boldsymbol{T}}^{\mathrm{T}} \boldsymbol{N} \boldsymbol{q} \mathrm{d} S
\end{aligned}
$$

The above formula takes the derivative of the interpolation variable $\alpha$ and considers the stationary value conditions to obtain:

$$
\frac{\partial \Pi_{\mathrm{HRS}}(\boldsymbol{q}, \boldsymbol{\alpha}, \boldsymbol{\beta})}{\partial \boldsymbol{\alpha}}=\int_{V} \boldsymbol{P}^{\mathrm{T}} \boldsymbol{C}[\boldsymbol{B} \boldsymbol{q}-\boldsymbol{P} \boldsymbol{\alpha}] \mathrm{d} V=0
$$

Or rewritten as

$$
H \alpha=G q
$$


Where

$$
\begin{aligned}
\boldsymbol{H} & =\int_{V} \boldsymbol{P}^{\mathrm{T}} \boldsymbol{C} \boldsymbol{P} \mathrm{d} V \\
\boldsymbol{G} & =\int_{V} \boldsymbol{P}^{\mathrm{T}} \boldsymbol{C B d} V
\end{aligned}
$$

From Eq. (39),

$$
\boldsymbol{\alpha}=\boldsymbol{H}^{-1} \boldsymbol{G} \boldsymbol{q}
$$

Substituting the above equation into Eq. (37) can transform the three-variable functional $\Pi_{\mathrm{HRS}}(\boldsymbol{q}, \boldsymbol{\alpha}, \boldsymbol{\beta})$ into the two-variable functional:

$$
\begin{aligned}
\Pi_{\mathrm{HRS}}(\boldsymbol{q}, \boldsymbol{\beta})= & \int_{V}\left[\left(\boldsymbol{P} \boldsymbol{H}^{-1} \boldsymbol{G} \boldsymbol{q}-\boldsymbol{R} \boldsymbol{\beta}-\boldsymbol{\varepsilon}^{\boldsymbol{p}}\right)^{\mathrm{T}} \boldsymbol{C}\left(\left(\boldsymbol{B}-\frac{1}{2} \boldsymbol{P} \boldsymbol{H}^{-1} \boldsymbol{G}\right) \boldsymbol{q}-\frac{1}{2}\left(\boldsymbol{R} \boldsymbol{\beta}+\boldsymbol{\varepsilon}^{\boldsymbol{p}}\right)\right)\right] \mathrm{d} V \\
& +\int_{V}\left(\boldsymbol{\sigma}^{0}\right)^{\mathrm{T}} \boldsymbol{R} \boldsymbol{\beta} \mathrm{d} V-\int_{V} \overline{\boldsymbol{f}}^{\mathrm{T}} \boldsymbol{N} \boldsymbol{q} \mathrm{d} V-\int_{S_{\sigma}} \overline{\boldsymbol{T}}^{\mathrm{T}} \boldsymbol{N} \boldsymbol{q} \mathrm{d} S
\end{aligned}
$$

Considering the stationary value conditions

$$
\left[\begin{array}{ll}
\boldsymbol{k}_{q q} & \boldsymbol{k}_{q \beta} \\
\boldsymbol{k}_{\beta q} & \boldsymbol{k}_{\beta \beta}
\end{array}\right]\left\{\begin{array}{c}
\boldsymbol{q} \\
\beta
\end{array}\right\}=\left\{\begin{array}{c}
\overline{\boldsymbol{F}} \\
\boldsymbol{F}^{0}
\end{array}\right\}+\left\{\begin{array}{c}
\boldsymbol{F}_{q p} \\
\boldsymbol{F}_{\beta p}
\end{array}\right\}
$$

where

$$
\begin{gathered}
\boldsymbol{k}_{\boldsymbol{q} \boldsymbol{q}}=\boldsymbol{G}^{\mathrm{T}} \boldsymbol{H}^{-1} \boldsymbol{G} \\
\boldsymbol{k}_{\boldsymbol{q} \boldsymbol{\beta}}=-\int_{V} \boldsymbol{B}^{\mathrm{T}} \boldsymbol{C} \boldsymbol{R} \mathrm{d} V \\
\boldsymbol{k}_{\beta \boldsymbol{q}}=-\int_{V} \boldsymbol{R}^{\mathrm{T}} \boldsymbol{C B} \mathrm{d} V=\left(\boldsymbol{k}_{\boldsymbol{q} \beta}\right)^{\mathrm{T}} \\
\boldsymbol{k}_{\boldsymbol{\beta} \boldsymbol{\beta}}=\int_{V} \boldsymbol{R}^{\mathrm{T}} \boldsymbol{C} \boldsymbol{R} \mathrm{d} V \\
\overline{\boldsymbol{F}}=\int_{V} \overline{\boldsymbol{f}}^{\mathrm{T}} \boldsymbol{N} \mathrm{d} V+\int_{S_{\sigma}} \overline{\boldsymbol{T}}^{\mathrm{T}} \boldsymbol{N} \mathrm{d} S \\
\boldsymbol{F}^{0}=-\int_{V} \boldsymbol{R}^{\mathrm{T}} \boldsymbol{\sigma}^{0} \mathrm{~d} V \\
\boldsymbol{F}_{\boldsymbol{q} \boldsymbol{p}}=\int_{V} \boldsymbol{B}^{\mathrm{T}} \boldsymbol{C} \boldsymbol{\varepsilon}^{\boldsymbol{p}} \mathrm{d} V \\
\boldsymbol{F}_{\boldsymbol{\beta} \boldsymbol{p}}=\int_{V} \boldsymbol{R}^{\mathrm{T}} \boldsymbol{C} \varepsilon^{\boldsymbol{p}} \mathrm{d} V
\end{gathered}
$$

The coefficient matrix in Eq. (44) has symmetry. In the right end item, $\overline{\boldsymbol{F}}$ represents the equivalent load of the specified external force (body force and surface force) corresponding to the nodal displacement $\boldsymbol{q}$, $\boldsymbol{F}^{0}$ represents the specified stress equivalent load corresponding to the unknown nonelastic strain interpolation parameter $\boldsymbol{\beta}, \boldsymbol{F}_{q \boldsymbol{p}}$ and $\boldsymbol{F}_{\boldsymbol{\beta} \boldsymbol{p}}$ correspond to the known non-elastic strain equivalent load $\boldsymbol{q}$ and $\boldsymbol{\beta}$, respectively. Observation formulas (46), (47) and (50) all have negative signs. If the non-elastic strain interpolation parameter $\boldsymbol{\beta}$ is contrary to the conventional strain signs, that is, when the interpolation matrix $\boldsymbol{R}$ has its own negative sign, the negative sign in the above formula can be avoided. 
Equation (44) can be expanded as

$$
\begin{gathered}
\boldsymbol{k}_{q q} \boldsymbol{q}=\overline{\boldsymbol{F}}-\boldsymbol{k}_{q \beta} \boldsymbol{\beta}+\boldsymbol{F}_{q p} \\
\boldsymbol{F}^{0}=\boldsymbol{k}_{\beta q} \boldsymbol{q}+\boldsymbol{k}_{\beta \beta} \beta-\boldsymbol{F}_{\beta p}
\end{gathered}
$$

Equation (53) is a conventional element balance equation without specified stress condition $(\boldsymbol{\beta}=0)$ or known $\boldsymbol{\beta}$ value; Eq. (54) is a method for calculating $\boldsymbol{F}^{0}$ using displacement and non-elastic parameters.

Although using Eq. (54) to solve $\boldsymbol{\beta}$ and then substituting Eq. (53) to eliminate $\boldsymbol{\beta}$ can reduce the order of Eq. (44), considering that the memory capacity of the current computer is large enough, in order to deal with the problem of partly known and partly unknown $\beta$, it is recommended to directly assemble the element according to formula (44). Since non-elastic parameters are associated to the stiffness matrix elements elementwisely, the use of sparse matrix technology can effectively reduce the storage and calculation amount.

After using formula (44) to assemble the overall balance equation of structure, $\boldsymbol{\beta}$ and $\boldsymbol{F}^{0}$ appear at both ends of the overall balance equation, and two solutions can be achieved:

(1) When the stress is specified, $\boldsymbol{F}^{0}$ is a known term, and the non-elastic parameter $\boldsymbol{\beta}$ that satisfies the specified stress condition can be obtained.

(2) When there is no specified stress condition, $\boldsymbol{\beta}=0$ (or other known non-elastic parameters) can be taken, which degenerates into a conventional finite element problem.

\section{Example analysis}

In order to verify and demonstrate the correctness of the method and the finite element formulation in this paper, the strain interpolation method provided by reference [2] is used

$$
\left\{\begin{array}{l}
\varepsilon_{\xi}=\alpha_{1}+\alpha_{2} \xi+\alpha_{3} \eta+\alpha_{4} \xi \eta+\alpha_{13} \xi \eta^{2} \\
\varepsilon_{\eta}=\alpha_{5}+\alpha_{6} \xi+\alpha_{7} \eta+\alpha_{8} \xi \eta+\alpha_{14} \xi^{2} \eta \\
\gamma_{\xi \eta}=\alpha_{9}+\alpha_{10} \xi+\alpha_{11} \eta+\alpha_{12} \xi \eta
\end{array}\right.
$$

The interpolation method of unknown non-elastic strain also adopts the same form as the total strain, namely $\boldsymbol{R}=\boldsymbol{P}$. A finite element program with specified stress conditions based on 9-node stress hybrid element (denoted as CMS9) is compiled, and two numerical examples are carried out.

Example 1:

A thin plate with uniform thickness $0.01 \mathrm{~m}$ is shown in the figure. It is divided into 24 elements. The material elastic modulus $E=2.4 \times 10^{10} \mathrm{~Pa}$, Poisson's ratio $v=0.2$, and the concentrated load applied at the right end midpoint is $P=\frac{E A}{480}=1.0 \times 10^{6} \mathrm{~N}$. The displacement boundary conditions are considered in two forms: the horizontal and vertical displacements of node 1-5 are constrained (Case 1); the horizontal displacement of node 1-5 is constrained, and the vertical displacement of node 3 is constrained (Case 2).

In Table 1, all non-elastic strain degrees of freedom are set as 0 , and the horizontal displacement of the load point which is normalized by PL/EA is exactly the same as the result in literature [2].

Table 1: Regularized horizontal displacements of loading points at cantilever end.

\begin{tabular}{lcc}
\hline Methods & CM [2] & CMS9 \\
\hline Case 1 & 1.2456 & 1.2456 \\
Case 2 & 1.2466 & 1.2466 \\
\hline
\end{tabular}




\section{Example 2:}

A notched thin plate with uniform thickness $0.01 \mathrm{~m}$ is shown in the figure. It is divided into 23 elements, and the material properties and loads are the same as in Example 1. The constraint method is selected as Case 2 of example 1: The horizontal displacement of nodes 1-5 is constrained, and the vertical displacement of node 3 is constrained. Four methods are used for comparison (all use $3 \times 3$ Gaussian integral).

(1) Method 1: For the grid in Figure 2, the plane 8-node element CPS8 of Abaqus software is used for calculation;

(2) Method 2: On the basis of the grid in Figure 2, each element is further refined into $2 \times 2$ elements, and the plane 8-node element CPS8 of the Abaqus software is used for calculation to obtain higher precision results;

(3) Method 3: For the grid in Figure 2, setting the non-elastic strain degrees of freedom of all elements as 0, CMS9 element is used;

(4) Method 4: The grid in Figure 1 is chosen and CMS9 element is used. Set $\boldsymbol{F}^{0}$ of element 6 is set as 0, and the stress components of the simulated element are 0 and cannot bear the load. At this moment, the non-elastic strain interpolation parameter of the element is set as an unknown quantity; the non-elastic strain interpolation parameters of the remaining elements are set as 0 .

The displacement results of the four methods are shown in Table 2.

It can be seen from the comparison in Table 2:

(1) The grid density of Method 1, Method 3 and Method 4 are the same. The result of CM9 unit used by Method 3 and Method 4 is closer to the high-precision solution of Method 2, that is, the accuracy of CM9 element is higher than that of CPS8 element;

(2) The results of Method 3 and Method 4 are exactly the same, indicating that by specifying the element's stress to be zero, it is possible to accurately simulate the absence of the element.

The position and serial number of the integration points of the element 18 in the grid of Figure 2 are shown in Figure 3, and the calculation results of the horizontal stress components of each integration point after being regularized by $\mathrm{P} / \mathrm{A}$ are shown in Table 3.

It can be seen from the stress comparison in Table 3.

(1) In the three methods, the element 18 is characterized by eccentric compression, which conforms to the actual force; Table 2 has verified that the results of Method 3 and Method 4 are more accurate than Method 1.

(2) The results of Method 3 and Method 4 are exactly the same, which shows once again: By specifying the element's stress to be 0 , it is possible to accurately simulate the absence of the element.

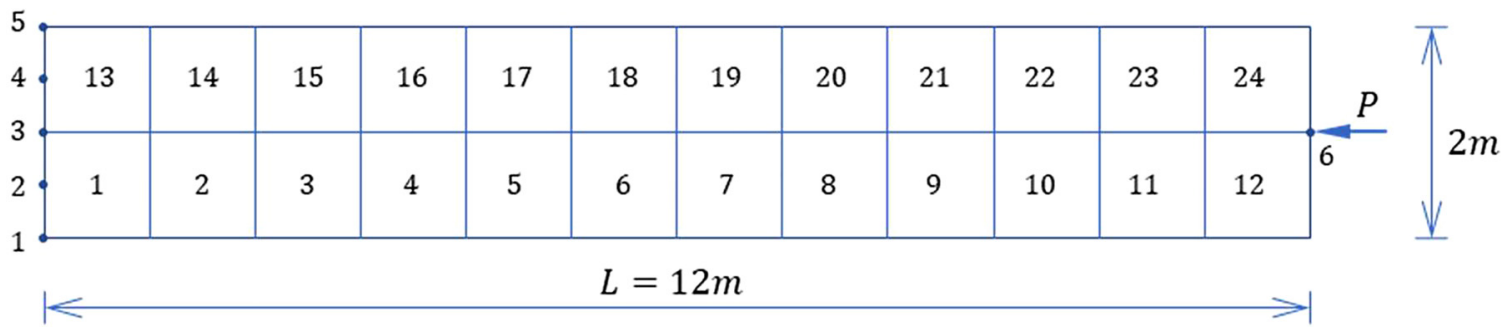

Figure 1: Rectangular thin plates are subject to concentrated force.

Table 2: Displacement of loading point at cantilever end.

\begin{tabular}{lrrrr}
\hline Methods & Method 1 & Method 2 & Method 3 & Method 4 \\
\hline$u$ & -0.05206 & -0.055992 & -0.0556662 & -0.0556662 \\
$v$ & -0.264289 & -0.28861 & -0.28286 & -0.28286 \\
\hline
\end{tabular}




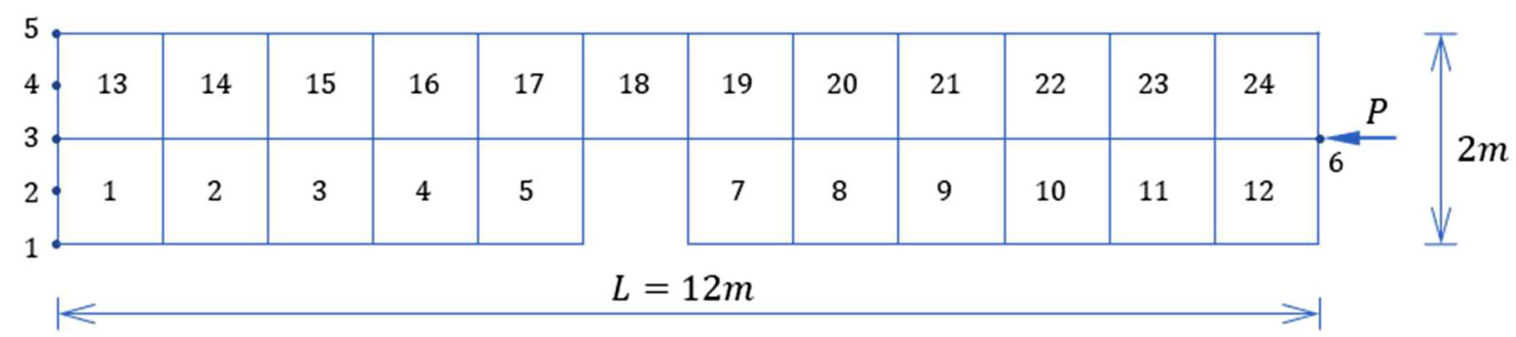

Figure 2: Concentrated force on a notched rectangular thin plate.

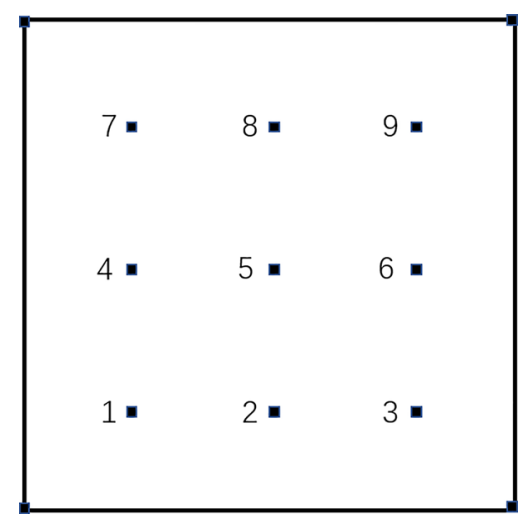

Figure 3: Position and ordinal number of Gaussian integral point of element 18.

Table 3: Regularized $\sigma_{x}$ at the Gaussian integral point of element 18.

\begin{tabular}{|c|c|c|c|c|c|c|c|c|c|}
\hline Location & 1 & 2 & 3 & 4 & 5 & 6 & 7 & 8 & 9 \\
\hline Method 1 & -3.4897 & -3.4587 & -3.4897 & -0.8236 & -0.7926 & -0.8236 & 1.1578 & 1.1889 & 1.1579 \\
\hline Method 3 & -3.3637 & -3.2739 & -3.3637 & -1.0 & -1.0 & -1.0 & 1.3637 & 1.2739 & 1.3637 \\
\hline Method 4 & -3.3637 & -3.2739 & -3.3637 & -1.0 & -1.0 & -1.0 & 1.3637 & 1.2739 & 1.3637 \\
\hline
\end{tabular}

\section{Conclusions}

Aiming at a class of specified stress problem in engineering, by introducing unknown non-elastic strains, the complementary energy functional is used to give(obtain) the elastic mechanics governing equations with specified stress conditions. Using Lagrange multiplier method, a modified complementary energy principle functional and virtual work equations with considering specified stress conditions are similarly deduced as the classical theorem. A functional based on the $\mathrm{H}-\mathrm{R}$ variational principle of conventional elastic mechanics, with displacement, stress and unknown non-elastic strain taking as independent variables, the Lagrange multiplier method is used to establish the $\mathrm{H}-\mathrm{R}$ variational functional satisfying the specified stress conditions; By replacing the stress with elastic strain, the variational functional with displacement, elastic strain and unknown non-elastic strain as independent variables is given, and the corresponding finite element formulation is derived based on a method of stress hybridization in the element.

Example 1 illustrated the validation of the algorithm proposed in this paper by comparison with the numerical calculation results of ABAQUS. Example 2 simulated the problem of a thin plate with gaps by specifying element stress of zero as life and death element technology. The numerical results of these two examples show the accuracy of the algorithm. Compared with the notched model, the life and death element technology with a specified stress of zero has the following advantages: (1) The modeling of the non-notch model is simple and easy; (2) The life and death element technology with a specified stress of zero can easily 
simulate the development of the gap. During the development of the gap, the geometric model does not need to be changed, and the total stiffness matrix remains unchanged. It is only necessary to specify the stress of the corresponding element as zero in the right end of the equation.

The specified stress problem proposed in this paper introduces the unknown non-elastic strain parameters into the governing equations of elastic mechanics, the corresponding complementary energy principle, the $\mathrm{H}-\mathrm{R}$ variational principle and its finite element formula, so that the non-elastic parameters and displacement appear as vectors in the degrees of freedom of the equilibrium equation. Stress appears in the right end of the balance equation, which can solve two types of problems with known non-elastic parameters and with specified stress conditions. The stress is adjusted by non-elastic strain parameters, which realizes the direct simulation of the specified conditions such as stress calculation and stress measurement. Using the obtained overall structural equilibrium equations, taking non-elastic parameters as design variables, it can also be used to establish optimization models based on stress and displacement fields.

Author contribution: All the authors have accepted responsibility for the entire content of this submitted manuscript and approved submission.

Research funding: None declared.

Conflict of interest statement: The authors declare no conflicts of interest regarding this article.

\section{References}

[1] T. H. H. Pian and C. C. Wu, Hybrid and Incompatible Finite Element methods[M], Boca Raton, FL, CRC Press, 2005.

[2] Z. Tian and T. H. H. Pian, Multivariable Variational Principles and Multivariable Finite Element methods[M], Beijing, Science Press, 2011, (in Chinese).

[3] L. I. Lei, C. Wu, and S. Xie, Optimization Method of Hybrid Element Stress Function for Strain Gradient Theory Based on Hellinger-Reissner Principle, vol. 37, Beijing, Acta Mechanica Sinica, 2005, pp. 301-306.

[4] F. Auricchio, G. Balduzzi, and C. Lovadina, "The dimensional reduction approach for 2D non-prismatic beam modelling: a solution based on Hellinger-Reissner principle[J]," Int. J. Solid Struct., vol. 63, pp. 264-276, 2015.

[5] F. Auricchio, G. Balduzzi, and C. Lovadina, "The dimensional reduction modelling approach for 3D beams: differential equations and finite-element solutions based on Hellinger-Reissner principle[J]," Int. J. Solid Struct., vol. 50, nos. 25-26, pp. 4184-4196, 2013.

[6] M. Rezaiee-Pajand and N. Gharaei-Moghaddam, "Force-based curved beam elements with open radial edge cracks[]]," Mech. Adv. Mater. Struct., vol. 27, no. 2, pp. 128-140, 2020.

[7] M. D’Ottavio and B. Kröplin, “An extension of Reissner mixed variational theorem to piezoelectric laminates[J]," Mech. Adv. Mater. Struct., vol. 13, no. 2, pp. 139-150, 2006.

[8] G. Altay and M. C. Dökmeci, "Variational principles for piezoelectric, thermopiezoelectric, and hygro-thermo-piezoelectric continua revisited[J]," Mech. Adv. Mater. Struct., vol. 14, no. 7, pp. 549-562, 2007.

[9] Y. Lee, K. Yoon, and P. S. Lee, “Improving the MITC3 shell finite element by using the Hellinger-Reissner principle[J]," Comput. Struct., vol. 110, pp. 93-106, 2012.

[10] A. F. Domínguez Alvarado and A. D. Diaz, “A stress approach model of moderately thick, homogeneous shells[]]," Math. Probl Eng., vol. 2018, pp. 1-16, 2018.

[11] A. F. Domínguez Alvarado and A. D. Díaz, "A mixed stress/displacement approach model of homogeneous shells for Elastodynamic problems[]]," Math. Probl Eng., vol. 2020, pp. 1-15, 2020.

[12] H. Sangtarash, H. G. Arab, M. R. Sohrabi, and M. R. Ghasemi, "A high-performance four-node flat shell element with drilling degrees of freedom[J],” Eng. Comput., vol. 34, pp. 1-16, 2020.

[13] J. Schröder, M. Igelbüscher, A. Schwarz, and G. Starke, “A Prange-Hellinger-Reissner type finite element formulation for small strain elasto-plasticity[]]," Comput. Methods Appl. Mech. Eng., vol. 317, no. C, pp. 400-418, 2017.

[14] N. Viebahn, K. Steeger, and J. Schröder, "A simple and efficient Hellinger-Reissner type mixed finite element for nearly incompressible elasticity[J],” Comput. Methods Appl. Mech. Eng., vol. 340, pp. 278-295, 2018.

[15] N. Viebahn, J. Schröder, and P. Wriggers, "A concept for the extension of the assumed stress finite element method to hyperelasticity[M]," in Novel Finite Element Technologies for Solids and Structures, Cham, Springer, 2020, pp. $107-126$.

[16] E. Artioli, S. de Miranda, C. Lovadina, and L. Patruno, "A family of virtual element methods for plane elasticity problems based on the Hellinger-Reissner principle[J]," Comput. Methods Appl. Mech. Eng., vol. 340, pp. 978-999, 2018.

[17] F. Dassi, C. Lovadina, and M. Visinoni, “A three-dimensional Hellinger-Reissner virtual element method for linear elasticity problems[]],” Comput. Methods Appl. Mech. Eng., vol. 364, p. 112910, 2020. 
[18] Y. Dong, Y. Ma, and W. Feng, “The hybrid nature element method for elasticity[J]," Chin. J. Theor. Appl. Mech., vol. 44, no. 003, pp. 568-575, 2012.

[19] Y. Ma and J. Xu, "Hybrid nature element method for the large deformation problem[J]," in China Mechanics Conference-2017 and the 60th Anniversary of the Chinese Society of Mechanics(C). (in Chinese).

[20] Y. Ma, Y. Zhou, Y. Dong, and W. Feng, "Hybrid natural element method for elastic large deformation problems[]]," Int. J. Appl. Mech., vol. 8, no. 04, p. 1650044, 2016.

[21] S. Yang, Y. Ma, and F. Wei, “A hybrid generalized element method based on H-R variational principle[J]," Appl. Math. Mech., vol. 34, no. 3, pp. 272-281, 2013.

[22] Y. Ding, C. Lai, L. I. Ning, and Z. LI, "Development and application of mixed element based on Hellinger-Reissner variation[J],”J. Tianjin Univ., vol. 48, no. 05, pp. 422-428, 2015.

[23] G. Qing and J. Tian, "Highly accurate symplectic element based on two variational principles[J]," Acta Mech. Sin., vol. 34, no. 01, pp. 151-161, 2018.

[24] Z. Zhao and G. Qing, “Improved noncompatible generalized mixed elements and performance analysis[]]," Appl. Math. Mech., vol. 40, no. 05, pp. 518-526, 2019.

[25] G. Qing and Y. Liu, "Convergence and precision of the dual-variable brick mixed element and its displacement element[J]," Appl. Math. Mech., vol. 38, no. 02, pp. 153-162, 2017.

[26] Y. Liu and L. I. Rui, "Parametered symplectic element and analysis of thermoelastic composite laminates[]]," Acta Mater. Compos. Sin., vol. 36, no. 5, pp. 1306-1312, 2019.

[27] T. Yao and Y. Yuzhe, "20-Node hexahedral symplectic elements for stress analysis of composite laminates[]]," Appl. Math. Mech., vol. 41, no. 05, pp. 509-516, 2020.

[28] J. Dujc and B. Brank, "Modeling fracture in elasto-plastic solids by embedded-discontinuity stress-hybrid finite element formulation[]]," Mech. Adv. Mater. Struct., pp. 1-17, 2020. https://doi.org/10.1080/15376494.2020.1786755.

[29] J. H. He, G. L. Liu, and W. B. Feng, "A generalized variational principle for coupled Thermoelasticity with finite displacement[]]," Commun. Nonlinear Sci. Numer. Simulat., vol. 3, no. 4, pp. 215-217, 1998.

[30] J. H. He, “Generalized Hellinger-Reissner principle[J]," ASME J. Appl. Mech., vol. 67, no. 2, pp. 326-331, 2000.

[31] A. A. Cannarozzi and F. Ubertini, “A mixed variational method for linear coupled thermoelastic analysis[]]," Int. J. Solid Struct., vol. 38, no. 4, pp. 717-739, 2001. 